\title{
Melanoma contains CD133 and ABCG2 positive cells with enhanced tumourigenic potential
}

\author{
Elena Monzani ${ }^{a}$, Floriana Facchetti ${ }^{a, h}$, Enrico Galmozzi ${ }^{a, h}$, Elena Corsini ${ }^{b}$, Anna Benettic, \\ Chiara Cavazzin ${ }^{d}$, Angela Gritti ${ }^{d}$, Andrea Piccinini ${ }^{e}$, Danilo Porro $^{f}$, Mario Santinami ${ }^{g}$, \\ Gloria Invernici ${ }^{b}$, Eugenio Parati ${ }^{b}$, Giulio Alessandri ${ }^{b}$, Caterina A.M. La Porta ${ }^{a, *}$
}

${ }^{a}$ Department of Biomolecular Science and Biotechnology, University of Milan, Milan, Italy

${ }^{\mathrm{b}}$ Istituto Neurologico Besta, Milan, Italy

${ }^{\mathrm{C} D e p a r t m e n t}$ of Anatomy Pathology, University of Brescia, Brescia, Italy

dInstitute for Stem Cell Research, Department of Biotechnology, San Raffaele Hospital, Milan, Italy

${ }^{\mathrm{e}}$ Istituto di Medicina Legale e delle Assicurazioni, Università degli Studi di Milano, Milan, Italy

${ }_{\mathrm{f}}^{\mathrm{f}}$ ipartimento di Biotecnologie e Bioscienze, Università degli Studi di Milano Bicocca, Milan, Italy

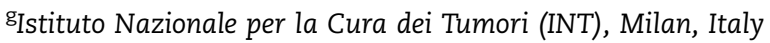

\section{A R T I C L E I N F O}

Article history:

Received 18 September 2006

Received in revised form

27 November 2006

Accepted 12 January 2007

Available online 22 February 2007

Keywords:

Melanoma

CD133

Cancer stem cells

\begin{abstract}
A B S T R A C T
The failure to eradicate most cancers and in particular melanoma may be as fundamental as a misidentification of the target. The identification of cancer stem/initiating cells within the tumour population with a crucial role for tumour formation may open new pharmacological perspectives. Our data show three main novelties for human melanoma: firstly, melanoma biopsy contains a subset of cells expressing CD133 (CD133+) and the latter is able to develop a Mart-1 positive tumour in NOD-SCID mice. Secondly, the WM115, a human melanoma cell line, has been found to express both CD133 and ABCG2 markers. This cell line grows as floating spheroids, expresses typical progenitors and mature neuronal/oligodendrocyte markers and is able to transdifferentiate into astrocytes or mesenchymal lineages under specific growth conditions. As in xenografts generated with CD133+ biopsy melanoma cells, those produced by the cell line displayed lower levels of CD133 and ABCG2. Thirdly, the WM115 cells express the most important angiogenic and lymphoangiogenic factors such as notch 4 , prox 1 and podoplanin which can cooperate in the development of the tumourigenic capability of melanoma in vivo.

Therefore, in this study, we demonstrate the presence of stem/initiating subsets in melanoma both in biopsy and in an established melanoma cell line grown in vitro and in xenografts. Interestingly, considering that melanoma gives metastasis primarily through lymphatic vessels, herein, we demonstrated that a melanoma cell line expresses typical lymphoangiogenic factors.
\end{abstract}

(ㄷ) 2007 Elsevier Ltd. All rights reserved.

\footnotetext{
* Corresponding author: Tel.: +39 2 50314927; fax: +39 250314932.

E-mail address: caterina.laporta@unimi.it (C.A.M. La Porta).

${ }^{h}$ E. Galmozzi and F. Facchetti made equal contributions to the paper. 0959-8049/\$ - see front matter @ 2007 Elsevier Ltd. All rights reserved. doi:10.1016/j.ejca.2007.01.017
} 


\section{Introduction}

The failure to eradicate most cancers may be as fundamental as a misidentification of the target. Our current therapies succeed at eliminating bulky disease and rapidly proliferating cells missing the tumour reservoir that is the source of disease recurrence and metastasis. For the last 30 years the idea that cancer may originate from immature/stem cells undergoing genetic mutations has been postulated. ${ }^{1}$ The idea that stem cells could be the origin of cancer cells was proposed on the basis of their practically unlimited capacity to proliferate and their self-renewal property. ${ }^{2}$ Thus, if cancer arises from a series of genetic mutations, stem cells may have more opportunity to accumulate mutations because of their long life-span. Cancer stem cells (CSCs) may represent only a minority of the cells within the tumour, however, they could be crucial for tumour formation. The involvement of cells displaying a stem cell phenotype in cancer formation and progression has been confirmed in acute myeloid leukaemia ${ }^{3,4}$ and breast cancer. ${ }^{5}$ More recently, a number of papers strongly support the idea that CSCs could be the basis for the origin of human brain tumours ${ }^{6,7}$ and prostate cancer. ${ }^{8}$

From the pharmacological perspective, the tumour stem/ initiating cell hypothesis provides several new insights into strategies for cancer treatment. In particular, cancer stem/initiating cells seem to retain the essential property of self-protection through the activity of multiple drug resistance transporters. Therefore, different malignancies may appear to be heterogeneous with respect to drug responsiveness. In fact, a cancer can respond to therapy initially but it may acquire drug resistance during the course of treatment. Other cancers appear intrinsically resistant. In this context, the cancer stem cell hypothesis considers that these cells have innate drug resistance by virtue of their capacity to remain quiescent.

Malignant melanoma is increasing in incidence in the US, Europe and Australia. ${ }^{9-11}$ Surgery remains the cornerstone of treatment for patients with loco-regional disease. However, while a few patients with advanced disease may be cured by surgery, the majority of patients die of their disease. ${ }^{12}$ In fact, cutaneous melanoma is among the most aggressive types of human cancer and, if untreated, virtually every melanoma has the potential to metastasise. Melanoma is defined as a neuroectodermal malignant tumour deriving from the transformation and the proliferation of melanocytes which, normally, reside in the basal cell layer of the epidermis. Recently, the gene expression analyses of cutaneous and uveal melanoma cells revealed that more aggressive tumour cells express genes associated with multiple phenotypes, including genes normally associated with neuronal, vascular and skeletal muscle and other precursor cell types. These findings seem to indicate that the more aggressive melanoma may contain deregulated cells with undifferentiated/stem cell-like phenotype. ${ }^{13-15}$ Consistent with this hypothesis is the recent observation that well established metastatic melanoma cell lines have been shown to exhibit multiple morphological, phenotypic, functional forms. ${ }^{16}$

In the present work we assess if melanoma tumours contain a subset of cells expressing genes, markers and stem/initiating cells-like properties. Basing the study on different reports that suggested CD133 as a marker of cancer/initiating cells in human glioblastoma, $, 6,17,18$ we first wanted to investigate the expression of CD133 marker in fresh tumour biopsies and in a continuous melanoma cell line (WM115) and then to characterise the CD133+ melanoma cell subset for tumourigenic potential, angiogenic and lymphoangiogenic properties. Secondly, since ABCG2, the second member of the $G$ family of $A B C$ transports, expression correlates with cancer cells with high tumourigenic potential, ${ }^{19,20}$ we investigated whether melanoma cells express ABCG2 both in vitro and in tumour xenografts.

Taken together, our findings demonstrate that, as in glioblastoma, human melanomas contain in vivo cancer stem/initiating cells with enhanced tumourigenic potential and that these cells can be identified by the expression of CD133, ABCG-2, angiogenic (notch 4) and lymphoangiogenic (prox1) markers.

\section{Materials and methods}

\subsection{Reagents}

Oil Red and isopropanol were obtained from Sigma-Aldrich (St. Louis, USA).

\subsection{Isolation of single melanoma cells from melanoma biopsy}

Melanoma biopsy (seven) was obtained from the National Italian Institute of Tumour of Milan, Italy, after received approval from the appropriate local Institutional Review Board. Melanoma metastatic specimens (around $1 \mathrm{~g}$ ) were obtained during surgical procedure. After several washes, tumour fragments were finely minced with scissors and then incubated for $2 \mathrm{~h}$ at $37^{\circ} \mathrm{C}$ in DMEM medium (Gibco, Grand Island, New York, USA) containing $0.2 \%$ of collagenase type I (Gibco) and $0.2 \%$ bovine serum albumine (BSA) (SIGMA). The tumour cell suspensions were washed in DMEM $+10 \%$ fetal bovine serum (FBS) (Gibco) and passed through a $100 \mu \mathrm{m}$ pore size filter to remove undigested tumour tissue macroaggregates. After centrifugation at $1000 \mathrm{~g}$, the pellet was resuspended in DMEM/F12 (Euroclone, Leeds, UK) medium optimised for growth of neural stem cells (NSC), ${ }^{2}$ containing $20 \mathrm{ng} / \mathrm{ml}$ EGF (Sigma), $20 \mathrm{ng} / \mathrm{ml} \mathrm{bFGF}$ (Boehringer Mannaheim Indianapolis IN, USA) $2 \mathrm{mmol} / \mathrm{L}$ glutamine, $0.6 \%$ glucose, $9.26 \mu \mathrm{g} / \mathrm{ml}$ putrescine, $6.3 \mathrm{ng} / \mathrm{ml}$ progesterone, $5.3 \mathrm{ng} / \mathrm{ml}$ sodium selenite, $25 \mu \mathrm{g} / \mathrm{ml}$ insulin, $100 \mu \mathrm{g} / \mathrm{ml}$ grade II transferrin sodium salt and $0.6 \% \mathrm{BSA}$ (all reagents were purchased from Sigma, St. Louis, MO, USA). After counting, cells were immediately used for further analysis.

\subsection{Isolation of $\mathrm{CD} 133$ positive cells biopsy}

Single melanoma cells were isolated from biopsy as described above as well as CD133+ cells using CD133 Cell Isolation Kit (Miltenyi Biotec SRL, Italy). An aliquot of cells were evaluated for purity by flow cytometry. Purities ranged from $70-80 \%$ for CD133+ (Mart-1+) and 95-98\% for CD133- (Mart-1+) cells and they were injected in NOD-SCID mice as described below. Furthermore, these cells were Mart-1 positive as described in the immunohistochemistry section. 


\subsection{Cell culture}

Human primary melanoma WM115 (ATCC CRL 1675) was cultured as in previous work. ${ }^{21}$ Since the genetic stability of cell lines under long-term culture conditions is not well defined, we performed the DNA fingerprinting assay on eight highly polymorphic microsatellite STR loci plus gender determination which has been found to be the best tool to screen the uniqueness of DNA profiles in a fingerprint database. ${ }^{22}$ The WM115 Short-tandem-repeat (STR) profiling (DNA fingerprinting) was performed using PowerPlex ${ }^{\circledR} 16$ System (Promega) on following STR loci: Amelogenin (X); CSF1PO (12); D13S317 (12,13); D16S539 (11,12); D5S818 (13); D7S820 (8); THO1 $(7,9)$; TPOX $(8,11)$; vWA $(15,1)$. The results in brackets showed the perfect match with ATCC profile. Human melanoma cell lines, IGR-37 (metastasis), IGR-39 (primary) were grown in DMEM medium supplemented with $15 \%$ foetal bo- vine serum and A375 (metastasis) in DMEM and 10\% foetal bovine serum.

\subsection{Animal experiments}

Five-week-old NOD-SCID mice were purchased from Charles River Laboratories. $5 \times 10^{5}$ WM115 cells were injected subcutaneously and after 60 days the tumour was isolated, treated with $0.2 \%$ of collagenase type I (Gibco) and $0.2 \%$ bovine serum albumine (BSA) (SIGMA) for 30 min shacking at $37^{\circ} \mathrm{C}$. After a brief centrifugation, the cells were prepared for FACS analysis while they were seeded for Western blot, RT-PCR or immunofluorescence analysis overnight (time zero). Biopsy sorted positive or negative CD133 cells $\left(3.5 \times 10^{5}\right)$ were injected subcutaneously in $200 \mu \mathrm{l}$ of phosphate buffered saline (PBS) and after 40 days the mice (four animals) were sacrificed,

Table 1 - Primers, annealing conditions and length of PGR products

\begin{tabular}{|c|c|c|c|c|}
\hline Gene & Primer sense & Primer reverse & $\begin{array}{l}\text { Fragment } \\
\text { size bp }\end{array}$ & $\begin{array}{l}\text { Annealing } \\
\text { temperature }\end{array}$ \\
\hline Notch 1 & 5'-GGTCAATGCGAGTGGC & 5'-GGCAGCAAGGCTACTGTG & 153 & $50^{\circ} \mathrm{C}$ \\
\hline Notch 2 & 5'-CGTTTAGTCAGGAATATGCGG & 5'-GGACACATTTATGTACCCAGAG & 146 & $58^{\circ} \mathrm{C}$ \\
\hline Notch 3 & 5'- GCTTCTCAGGTCCTCGCTGT & 5'-GGCACAGTGACAGGTGAAGG & 124 & $60^{\circ} \mathrm{C}$ \\
\hline Notch 4 & 5'-TCTGGGGCCCGGGCTGAAGAAAG & 5'-ACGCCGGATGAGCTGGAGGACGAGA & 393 & $66^{\circ} \mathrm{C}$ \\
\hline Jagged 1 & 5'-ACCTTTAACAAGGAGATGATG & 5'-CTCAATTCACTGCAAATGTGC & 62 & $56^{\circ} \mathrm{C}$ \\
\hline Jagged 2 & 5'-CTCCATCGATGGACTTCAGC & 5'-CACATTCCGTTTGATAGTGG & 131 & $56^{\circ} \mathrm{C}$ \\
\hline$\Delta \mathrm{LL} 1$ & 5'-AACAAGAAAACСССТСТССТСАС & 5'-CTCTGCGGAACTCCAAGAATCTA & 318 & $63^{\circ} \mathrm{C}$ \\
\hline$\Delta \mathrm{LL} 3$ & 5'-GCGTGGAAAGGGATGAATGC & 5'-TTTCGCTGGCAGGGTTAGGC & 465 & $58^{\circ} \mathrm{C}$ \\
\hline$\Delta \mathrm{LL} 4$ & 5'-CTGGGTGCGTCTCTTGCT & 5'-AAGGGCCTAGACCAGAGAGC & 122 & $56^{\circ} \mathrm{C}$ \\
\hline Sox 18 & 5'-ACCСTTCCTGGGCTTCTG & 5'-AAAAGGAGCAGGTGCTTCAA & 153 & $55^{\circ} \mathrm{C}$ \\
\hline endogline & 5'-AGCAGGAGAGACTGAGCAGC & 5'-CAAGTGGTCTGTCTCCTGGG & 236 & $60^{\circ} \mathrm{C}$ \\
\hline Semaphorine $3 \mathrm{~A}$ & 5'-CCCTGGAAGTCATTGACACAG & 5'-GGTATGTCCTGGCCTTTGCCG & 230 & $60^{\circ} \mathrm{C}$ \\
\hline Semaphorine 3F & 5'-GGCAGCCTAGCCCTTGTC & 5'-TGGTTAGGCAGGCTCTGTTT & 210 & $55^{\circ} \mathrm{C}$ \\
\hline podoplanin & 5'-CCAGGAACCAGCGAAGACC & 5'-GCGTGGACTGTGCTTTCTGA & 119 & $57^{\circ} \mathrm{C}$ \\
\hline PROX-1 & 5'-TTCAGATGGAGAAGTACGCA & 5'-GGACTGCTACTCTTCATACA & 360 & $53^{\circ} \mathrm{C}$ \\
\hline VEGFR3 & 5'-AGCCATTCATCAACAAGCCT & 5'-GGCAACAGCTGGATGTCATA & 298 & $53^{\circ} \mathrm{C}$ \\
\hline CD133 & 5'-TACCAAGGACAAGGCGTTCAC & 5'-CAGTCGTGGTTTGGCGTTGTA & 470 & $59^{\circ} \mathrm{C}$ \\
\hline adipsin & 5'-GGTCACCCAAGCAACAAAGT & 5'-CCTCCTGCGTTCAAGTCATC & 272 & $56^{\circ} \mathrm{C}$ \\
\hline ABCG2 & 5'-CCAGTTCCATGGCACTGGCCATA & 5'-CAGGGCCACATGATTCTTCCACA & 347 & $60^{\circ} \mathrm{C}$ \\
\hline ABCB1 & 5'-GCAAAGCTGGAGAGATCCTCACCA & 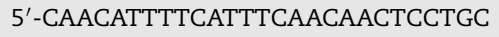 & 305 & $60^{\circ} \mathrm{C}$ \\
\hline GAPDH & 5'- TCTAGACGGCAGGTCAGGTCCACC & 5'-CCACCCATGGCAAATTCCATGGCA & 598 & $58^{\circ} \mathrm{C}$ \\
\hline VEGF & 5'-GGCCAGCACATAGGAGAG & 5'-TGCAGGAACATTTACACG & 157 & $50^{\circ} \mathrm{C}$ \\
\hline Nested primers for VEGF & 5'-CAGCACATAGGAGAG & 5'-TGCAGGAACATTTACACG & 154 & $50^{\circ} \mathrm{C}$ \\
\hline Flt-1 & 5'-TTTTACCGAATGCCACCTC & 5'GCGTGCTAGCTGGATGTCTT & 159 & $58^{\circ} \mathrm{C}$ \\
\hline KDR & 5'-GAACCAAATTATCTCСАТСТT & 5'-GCACTCCAATCTCTATCAGC & 180 & $58^{\circ} \mathrm{C}$ \\
\hline Nested primers for KDR & 5'-TGTGGCATCTGAAGGCTCAA & 5'-GCACTCCAATCTCTATCAGC & 125 & $58^{\circ} \mathrm{C}$ \\
\hline Ang-1 & 5'-ACCGAGCCTATTCACAGTAT & 5'-CAAGCATCAAACCACCATCCT & 180 & $55^{\circ} \mathrm{C}$ \\
\hline Nested primers for Ang-1 & 5'-CGAGCCTATTCACAGTAT & 5'-CAAGCATCAAACCACСATCCT & 178 & $55^{\circ} \mathrm{C}$ \\
\hline Ang-2 & 5'-TTCTAAACATCCCAGTCCAC & 5'-CCCGTCAGCACCGAGCACAC & 132 & $50^{\circ} \mathrm{C}$ \\
\hline Nested primers for Ang-2 & 5'-TCTAAACATCCCAGTCCAC & 5'-CCCGTCAGCACCGAGCACAC & 131 & $50^{\circ} \mathrm{C}$ \\
\hline Tie-2 & 5'-GTCCCGAGGTCAAGAGGTG & 5'-CAAGTCATCCCGCAGTAGG & 177 & $60^{\circ} \mathrm{C}$ \\
\hline CD31 & 5'-AGTGGTTATCATCGGAGTG & 5'-TCATTTATTGGTTTCATT & 222 & $50^{\circ} \mathrm{C}$ \\
\hline Nested primers for CD31 & 5'-AGTGGTTATCATCGGAGTG & 5'-CTGCTGGCCTGGACATTTC & 110 & $50^{\circ} \mathrm{C}$ \\
\hline Tie-1 & 5'-GCCATGATCAAGAAGGACGG & 5'-GTTCTCTCCGACCAGCACAT & 407 & $55^{\circ} \mathrm{C}$ \\
\hline Neuropilin 1 & 5'-ATGGAGAGGGGGCTGCCG & 5'-CAACATCAGGGAATCCATCCC & 659 & $55^{\circ} \mathrm{C}$ \\
\hline Neuropilin 2 & 5'-ATGGATATGTTTCCTCTCACC & 5'-CTGGAGATACTCCTTGTTGG & 989 & $55^{\circ} \mathrm{C}$ \\
\hline E-Cadherin & 5'-GCCAAGCAGCAGTACATTCTACACG & 5'-GCTGTTCTTCACGTGCTCAAAATC & 341 & $68^{\circ} \mathrm{C}$ \\
\hline N-Cadherin & 5'-GTGCCATTAGCCAAGGGAATCAG & 5'-GCGTTCCTGTTCCACTCATAGAG & 369 & $68^{\circ} \mathrm{C}$ \\
\hline P-Cadherin & 5'-GACCAACGAGGCCCCTTTTGTGCG & 5'-GTGGTGGGGGGCTTCC TTGTCCA & 356 & $68^{\circ} \mathrm{C}$ \\
\hline Eph-A2 & 5'-CTCACACACCCGTATGGCAA & 5'-GGCTCTCAGATGCCTCAAAC & 736 & $60^{\circ} \mathrm{C}$ \\
\hline
\end{tabular}


photographed and tumours were fixed in $10 \%$ formalin for immunohistochemistry.

\subsection{Cytofluorimetric analysis}

Subconfluent cells were fixed in iced ethanol at $4{ }^{\circ} \mathrm{C}$. Cells were stained with a monoclonal antibody anti CD133 PE-conjugated, clone 2 (Miltenyi Biotec S.r.l., Italy; this antibody does not cross-react with mice) or anti-CD133, epitope 2 (CD133/2) (Miltenyi Biotech) or anti-ABCG2-FITC conjugated (Chemicon). We have also carried out experiments fixing after staining and we obtained the same results. Data were acquired with a FACSscanplus (Becton-Dickinson) equipped with an argon-ion laser (excitation wavelength $488 \mathrm{~nm}$, laser power $200 \mathrm{~mW}$ ) (500-600 cells/s). Typically 30,000 cells were analysed per sample. DNA deconvolutions were carried out using the software program CELLQUEST (Becton-Dickinson). The experiments were repeated at least four times. For CD133/2, non specific staining was evaluated using a monoclonal antibody for IgG1 and IgG2a (Simultest control, Becton Dickinson, CA).

\subsection{Differentiation assays of melanoma cells}

Melanoma cells were induced to differentiate towards different mesenchimal lineages such as adipogenic according to Barbero et al. ${ }^{10}$ After differentiation, cells were incubated in Oil Red O solution ( $0.6 \%$ in $60 \%$ isopronanol) for $25 \mathrm{~min}$ and counterstained with haematoxylin for $10 \mathrm{~s}$. The extent of adi- pogenic differentiation was determined by counting the number of positively stained cells in 24 independent microscope fields (total area analysed in each experimental group $=40 \mathrm{~mm}^{2}$ ). The results are reported by the mean \pm standard differentiation (SD). Part of differentiated cells was used for the analysis of adipsin by RT-PCR.

\subsection{Immunofluorescence and immunohistochemistry analysis}

For immunofluorescence analysis, the cells were fixed in $4 \%$ paraformaldeide rinsed with phosphate-buffered saline (PBS) and incubated for $90 \mathrm{~min}$ at $37{ }^{\circ} \mathrm{C}$ in PBS containing $10 \%$ normal goat serum, $0.3 \%$ Triton-X100 (omitted when antibodies against membrane-bound antigens were used) and appropriate primary antibodies for $20 \mathrm{~min}$ : anti- $\beta$ tubulin type III (Tuj1; 1:500; Babco), anti-microtubule-associated-protein 2 (MAP2) (1:300, Immunological Science), anti-nestin (1:200, Chemicon), anti-PSA-NCAM (1:500, Produits Valbiotec), antiA2B5 (1:1000, Chemicon), anti-O4 (1:100, Chemicon), antiGFAP (1:300; Dako, Carpinteria, CA), anti-GalC (1:100, Chemicon), anti-Ki67 (1:1000, Novocastra). For anti-CD133 (1:100, Abcam; this antibody cross-reacts with mice and humans), an a $2 \mathrm{~h}$ incubation at $4{ }^{\circ} \mathrm{C}$ was carried out. Then, the cells were incubated with the suitable anti-mouse immunoglobulin $\mathrm{G}$ (IgG) (1:300 Cy3; Jackson Immunoresearch) for $45 \mathrm{~min}$. Regarding CD133, the cells were incubated with goat anti-rabbit Alexa 488 (1:700; Molecular Probes), and the nuclei were stained with




$100 \mathrm{X}$



$400 x$

Fig. 1 - Expression of CD133 in human melanoma biopsies (seven) and tumour-initiating capability of CD133+ cells in NOD-SCID mice. (A) A representative flow cytometry analysis. Melanoma specimens were incubated for $2 \mathrm{~h}$ in medium containing $\mathbf{0 . 2} \%$ of collagenase type I and single cells were fixed in iced ethanol and stained with anti CD133 PE-conjugated. The dot-plot shows the percentage of CD133+ cells less than 1\%. (B) CD133+ cells were obtained by beads sorting (CD133 Cell Isolation Kit) from melanoma biopsies. One hundred thousand cells were injected subcutaneously into NOD-SCID mice (four). Detectable tumour appeared 40 days later. Photography shows a representative size tumour obtained. Immunohistochemistry of Mart1 was performed to assess the melanocytic origin of the tumour growth in NOD-SCID mice. The injection of CD133- cells did not give a detectable tumour. 
DAPI. For negative control, the cells were incubated with the suitable anti-immunoglobulin G: anti-mouse immunoglobulin G (IgG) (1:300, Cy3; Jackson Immunoresearch) for $45 \mathrm{~min}$. For rodhamine uptake, WM115 cells were grown in $9.6 \mathrm{~cm}^{2}$ culture dishes in DMEM containing 2\% FBS. Rhodamine $123(0.1 \mu \mathrm{g} / \mathrm{ml}$, Sigma Aldrich) was added directly to each culture dish, and the cells were incubated for $20 \mathrm{~min}$ at $37^{\circ} \mathrm{C}$. After rhodamine staining, the cells were fixed in $4 \%$ paraformaldehyde for 20 min at $4{ }^{\circ} \mathrm{C}$. The nuclei were stained with DAPI.

For immunohistochemistry, the cells were fixed in 95\% ethanol and the antigens were unmasked by means of heating in a microwave oven at $750 \mathrm{w}$ in $10 \mathrm{nmol} / \mathrm{L}$ citric acid buffer ( $\mathrm{pH}$ 6.0) for $5 \mathrm{~min}$. The cells were incubated with the specific antibody Mart-1 (DAKO, 1:40), VEGFR-3 (kind gift of Alitalo, 1:100), LYVE-1 (Alexis, 1:100), prox-1 (Alexis, 1:100) or D2-40 (Signet, 1:160), overnight at $4^{\circ} \mathrm{C}$. Then, the cells were treated with biotinylated secondary antibodies and the avidin-biotin peroxidase complex kit (Dako Cytomation A/S, Glostrup, Denmark).

\subsection{Western blot}

Fifty micrograms of proteins were run in 10\% SDS-PAGE and transferred on PVDF sheet. ${ }^{23}$ The blots were incubated for 1-
$2 \mathrm{~h}$ in blocking solution (5\% skimmed milk in Tris-buffer), then for $1 \mathrm{~h}$ with the following primary antibodies: antiCD133 $(2 \mu \mathrm{g} / \mathrm{ml})$ (Abcam); anti-podoplanin $(2 \mu \mathrm{g} / \mathrm{ml})$ (ReliaTech, Braunschwaig, Germany); beta-actin, (1:5000) (Sigma). Next the sheet was incubated for $1 \mathrm{~h}$ with HRP-conjugated secondary antibodies (Amersham Pharmacia Biotech) against mouse or rabbit immunoglobulins. The bands were visualised using the ECL-Plus detection system (Amersham Pharmacia Biotech).

\subsection{RT-PCR analysis}

Total cellular RNA was extracted from human cell lines using the RNeasy Mini Kit (Qiagen) and complementary DNA (cDNA) was synthesised using 3-5 $\mu$ g of total RNA (eAMV-RT, Sigma); $2 \mu \mathrm{l}$ of cDNA was used for PCR analysis according to Table 1 and for nested PCR, $2 \mu$ l of the first product was amplified using the primers shown (Table 1). During RNA preparation, to exclude potential genomic DNA contamination, samples were first treated with RNase-free DNase I and then analysed using RT-PCR. GAPDH was used as housekeeping gene. Finally, to check the specificity of the amplification, all the bands were purified and sequenced.

A
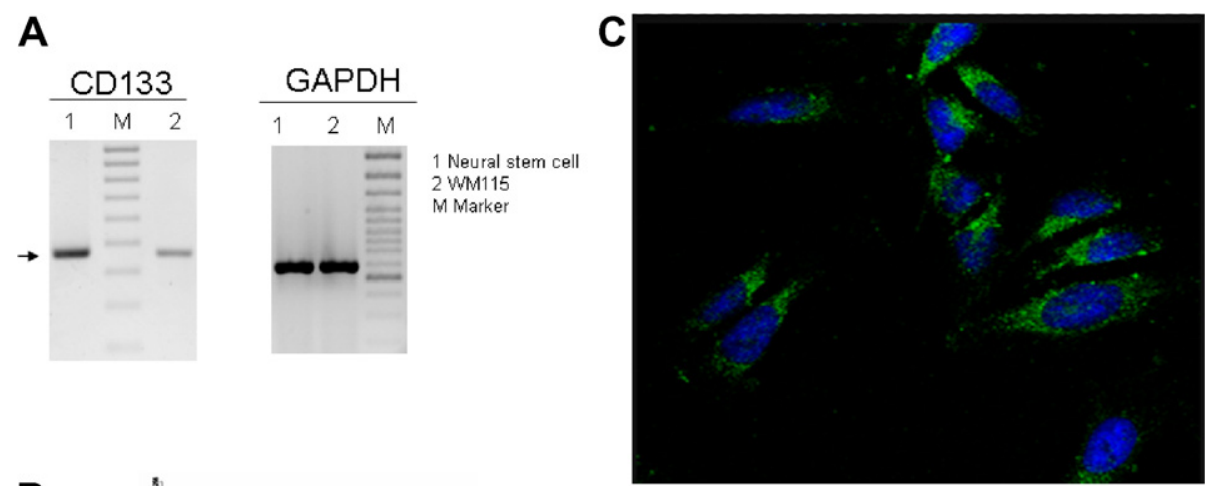

B

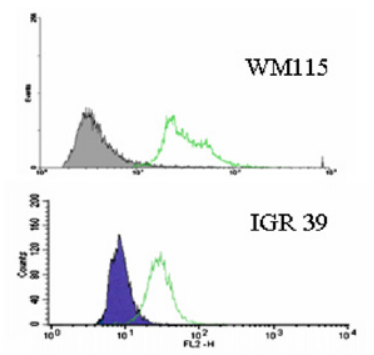

Caterina.A.M. La Porta et al.
D

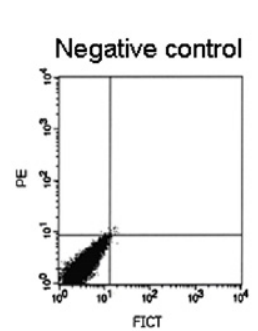

CD133-PE

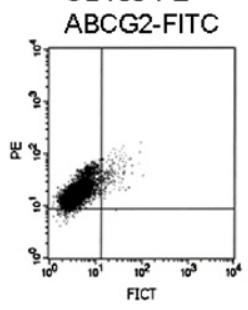

Fig. 2 - Expression of CD133 in WM115 human melanoma cell line. All the panels show a representative result out of four carried out. (A) Total RNA was extracted from WM115 cells using the RNeasy Mini Kit. To exclude potential genomic DNA contamination, samples were treated with RNase-free DNase I and then complementary DNA (cDNA) was synthesised. As positive control, human neural stem cells were used according to Cavazzini et al. ${ }^{36}$ (lane 1). A band of 470 bp was amplified and then sequenced. GAPDH was used as housekeeping gene. (B) Flow cytometry of CD133 expression in WM115 and IGR 39. Cells were fixed in iced ethanol stained with anti CD133 PE-conjugated (Mylteni). WM115 cells express CD133 (green line). The gray line shows the autoflorescence of the cells. (C) Cells were fixed with $4 \%$ paraformaldehyde for 20 min and then stained with anti-CD133 $2 \mathrm{~h}$ (1:100, Abcam). The nuclei were stained with DAPI. (D) Flow cytometry of CD133 and ABCG2 expression in WM115 cells. The latter were stained with anti-CD133-PE (Mylteni) conjugated and anti-ABCG2-FITC conjugated (Chemicon). No specific staining was evaluated using a monoclonal antibody for IgG1 and IgG2a (negative control). 


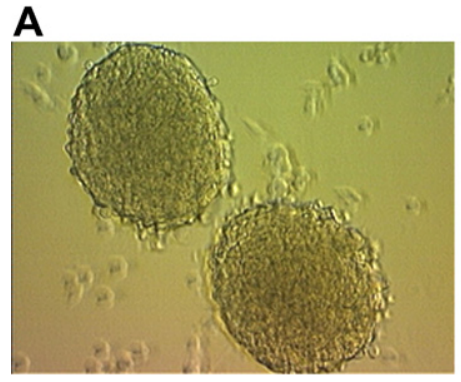

B

\begin{tabular}{|l|l|}
\hline & WM115 \\
\hline$\beta$ tubulin III & + \\
\hline MAP2 ${ }^{C}$ & + \\
\hline GFAP & - \\
\hline Gal-C & + \\
\hline O4 & - \\
\hline A2B5 & + \\
\hline Nestin & - \\
\hline PSA-NCAM & - \\
\hline Ki67 & + \\
\hline CD133 (green)/ \\
MAP-2(red)
\end{tabular}

C

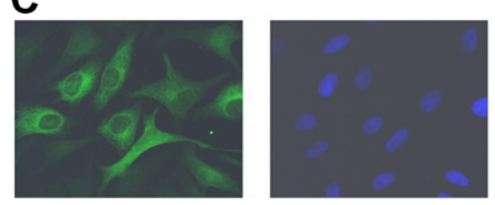

$\beta$-tubulin type III
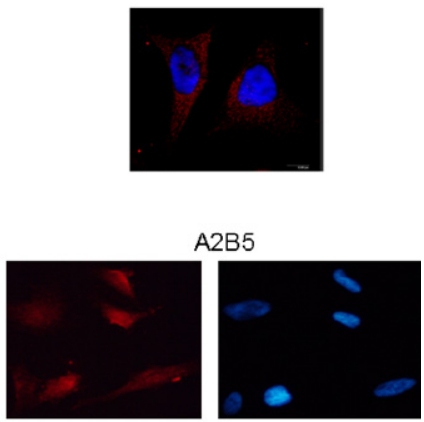

GalC

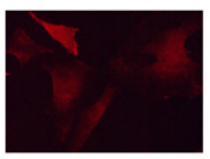

Fig. 3 - The stem/initiating phenotype of WM115 cells. (A) WM115 cells grown in standard medium as spheroids under non adherent plate (Nunc). Herein is shown a typical example. (B) Immunofluorescence of beta-tubulin type III, A2B5 and GalC in WM115 cells. The cells were fixed with 4\% paraformaldehyde and incubated with anti-tubulin type III (Tuj1; 1:500), anti A2B5 (1:1000) or anti-GalC (1:100) overnight at $4^{\circ} \mathrm{C}$. Then the cells were incubated with the suitable anti-mouse immunoglobulin (IgG) (1:300) for 45 min. DAPI was used to stain nuclei. Double staining between MAP2 (red, 1:300, Immunological Science) and CD133 (green; 1:100 Abcam) overnight showed that all the cells are positive for both markers (beta-tubulin gave the same results, not shown). (C). The cells were grown in DMEM/F12 medium in the absence of EGF for $72 \mathrm{~h}$ and then for an additional 6 days in medium without EGF and FGF-2 but with $2 \%$ foetal bovine serum (FBS). Then, the cells were fixed with $4 \%$ paraformaldehyde and then incubated with anti-GFAP (1:300) overnight at $4{ }^{\circ} \mathrm{C}$. Nuclei are stained with DAPI.

\section{Results}

\subsection{Fresh CD133+ cells isolated from melanoma} specimens demonstrate tumour-initiating property in vivo

In order to investigate the possible presence of a stem/initiating cell population in melanoma, we first analysed the expression of CD133 in seven human melanoma specimens. Briefly, fresh surgical biopsy was mechanically/enzymatically disaggregated and the resulting single cells suspension was analysed by FACS. As shown in Fig. 1 (panel A), we have found less than $1 \%(0.5 ; 0.8 ; 0.3 ; 0.8 ; 0.2 ; 0.3 ; 0.2 \%$ for each biopsy, respectively) of cells positive for CD133. In accordance with the idea that cancer stem/initiating cells may represent only a small percentage of the tumour cell population, we then tested the tumourigenic capacity of CD133+ melanoma cells. Thus, upon magnetic beads selection, the sorted CD133+ or CD133- cells were injected subcutaneously into 5-week old NOD-SCID mice. After 40-50 days, the mice injected with CD133+ cells developed a detectable tumour (Fig. 1, panel B, $0.2 \mathrm{~g}$ ), whereas mice injected with CD133- melanoma cells did not develop a tumour even 4 months after injection. This result was obtained in all the metastatic melanoma specimens processed. To confirm that tumours originating in mice were melanomas, the animals were sacrificed and histology of the tumour was performed. As shown in Fig. 1 (panel B), the expression of Mart-1, a typical melanocyte marker, was detected before and after the injection in NOD-SCID mice.

3.2. WM115 human melanoma cell line contains cells with cancer stem/initiating-like properties and expresses markers of stem/progenitor cells

Important in vivo results seem to demonstrate that melanoma behaves like other neuroectodermal tumours ${ }^{6,7}$ and only a minor fraction of CD133+ tumour cells was able to recapitulate the original tumour. Therefore, to bypass the problem that there is a low number of cells CD133+ in biopsy and that long-time in culture can alter the expression of pivotal cellular markers and, more importantly, can alter their biological behaviour, we used a well established, tumourigenic and genetic stable melanoma cell line, WM115, extensively studied by our group ${ }^{21}$ for further studies on stem cell properties of melanoma. Since the genetic stability of cell line under 




Fig. 4 - Differentiation of WM115 cells into mesenchymal lineage. (A) Subconfluent cells were treated with adipogenic induction medium (see Section 2) for $72 \mathrm{~h}$ and then with adipogenic maintenance medium (see Section 2 ) for $24 \mathrm{~h}$. WM115 cells incorporate Oil Red after differentiation (see arrows). The extent of adipogenic differentiation was determined by counting the number of positively Oil Red stained cells in 24 independent microscope fields. In the histograms are reported the mean \pm SD. ${ }^{*} p<0.001$ versus undifferentiated WM115 cells. (B) Level of expression of adipsin by RT-PCR. Total RNA was extracted from WM115 cells using the RNeasy Mini Kit. To exclude potential genomic DNA contamination, samples were treated with RNase-free DNase I and then complementary DNA (cDNA) was synthesised. A band of 272 bp was amplified and then sequenced. GAPDH was used as housekeeping gene. The ratio between the densitometric values of adipsin of undifferentiated and differentiated cells with respect to housekeeping gene show an increase in the level of expression of adipsin after differentiation (1.5-fold).

long-term culture conditions is not well defined, we performed the DNA fingerprinting assay on eight highly polymorphic microsatellite STR loci plus gender determination demonstrating a perfect match with ATCC profile (see Section 2). We first investigated for the presence of CD133. As shown in Fig. 2, we found that practically all WM115 cells expressed CD133 by means of FACS analysis, immunofluorescence and RT-PCR. We have also analysed other melanoma cell lines (A375, IGR 32 and IGR39), all positive for CD133 (Fig. 2 shows IGR 39). CD133+ cells were also positive for ABCG2 (Fig. 2, 3\%). However, we have chosen to use as model, WM115 cells, since we have previously characterised such a cell line and they are tumourigenic in vivo. ${ }^{21}$ The stem/progenitor phenotype of this cell line was confirmed by its capacity to survive and grow as floating spheroids when cultured in a serum free medium or in a non-adherent plate (Fig. 3, panel C) and by the analysis of neurogenic markers. Basically, WM115 cells positive for CD133 expressed markers of neural ( $\beta$-tubulin type III and MAP2), but not of their precursors (nestin and polysialic neural cell adhesion molecule) whereas they expressed markers of oligodendrocytes and of their precursors (GalC and A2B5) (Fig. 3). In contrast, GFAP (presumed to be expressed by differentiated astrocytes) and antigen $\mathrm{O} 4$ (expressed in pre-oligodendrocytes) were undetectable (immunofluorescence, data not shown). However, when the cells were maintained in a differentiating medium like that for neural stem cells, ${ }^{24}$ they started expressing GFAP, without any significant morphological differences (Fig. 3, panel B).

Furthermore, since melanocyte are derived from the neural crest during embryonic development and the transient neural crest consists of pluripotent stem cells that give rise to a wide array of lineages including cephalic mesenchyme, we have investigated whether WM115 can differentiate into mesenchymal lineages such as adipogenic. The extent of adipogenic differentiation was determined by counting the number of positive Oil Red stained cells. WM115 did not stain for Oil Red, however, after culture conditions that sustained adipogenic differentiation, more than $30 \%$ of the cells acquired positivity for Oil Red (Fig. 4, panel A). These cells are also negative for CD133 (not shown). RT-PCR of adipsin, a typical adipogenic marker, was carried out to confirm adipogenic differentiation. As shown in Fig. 4 (panel B) a substantial increment (1.5-fold versus control) of adipsin expression was observed. 
3.3. WM115 cells express angiogenic and lymphoangiogenic markers

An essential feature of cancer stem/initiating cells is their capacity to express vasculogenic-angiogenic properties in order to establish a tumour in vivo. To this end we analysed the expression of the pivotal factors involved in vasculogenesis/ angiogenesis by RT-PCR. In Table 2 are reported all the factors expressed by WM115 cells. Interestingly WM115 cells express stem cell-like factors such as notch 4 and many other factors linked to vasculogenesis/angiogenesis. Since melanoma uses lymphatic vessels to metastasise, we have analysed whether WM115 cells express typical markers of lymphatic endothelium such as VEGFR-3, LYVE-1, prox1 and podoplanin. As shown in Fig. 5, VEGF-R3, LYVE1, prox1 and podoplanin were expressed in WM115 cells where no signal has been detected for D2-40. The magnification for D2-40 (20 and 100X) was chosen to emphasise that all WM115 cells do not express this marker. In addition, since podoplanin showed faint levels by immunohistochemistry, Western blot analysis was also carried out (Fig. 5).

\subsection{Expression of CD133 and ABCG2 in WM115 cells grown in vivo in NOD-SCID mice}

We wanted to investigate the levels of CD133 and ABCG2 in tumour xenograft obtained by injecting WM115 cells in NOD-SCID mice. In fact, cell lines maintained in vitro often
Table 2 - Summary of angiogenic/vasculogenic factors as well as adhesion and stem cell markers expressed by WM115 cells

Gene

Expressed (+)

\section{VEGF}

FLt-1

Flk-1

Nrp-1

Nrp-2

Ang-1

Ang-2

Tie-1

Tie-2

Ephrine A2

CD31

Endogline

Notch 4

JAG1

JAG2

DLL1

DLL3

DLL4

Sox1 8

Semaphorine 3A

Semaphorine 3F

E-cadherin

$\mathrm{N}$-cadherin

P-cadherin

ABCG2

ABCB1
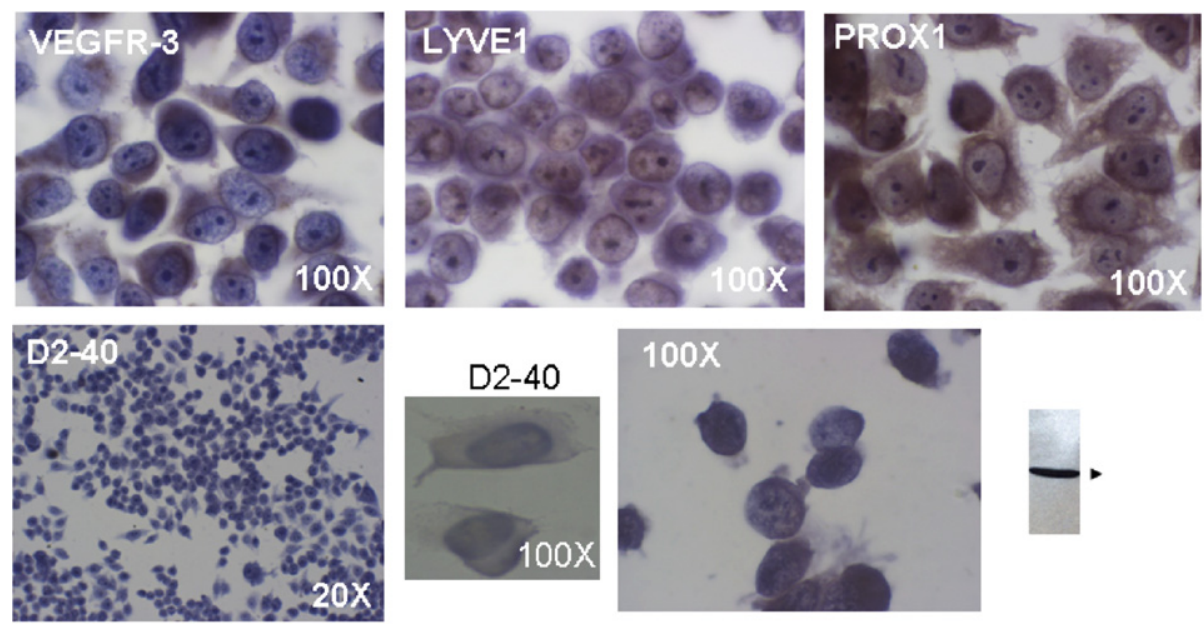

podoplanin

\begin{tabular}{lc}
\hline & WM115 \\
\hline VEGFR3 & + \\
Podoplanin & + \\
PROX1 & ++ \\
D2-40 & - \\
LYVE1 & + \\
\hline
\end{tabular}

Fig. 5 - Expression of typical lymphoangiogenic markers. Immunohistochemistry of VEGF-R3, D2-40, prox1, podoplanin and LYVE-1 in WM115 human melanoma cells fixed in 95\% ethanol and processed with the standard avidin-biotin peroxidase complex. DAB was used as chromogen. Due to the low level of expression of podoplanin by immunohistochemistry, Western blot analysis was also carried out. $50 \mu \mathrm{g}$ of protein was loaded on $10 \%$ SDS-PAGE and complex antigen-antibody was detected with ECL system. WM115 cells express VEGFR-3, prox-1, podoplanin, LYVE-1 while D2-40 is undetectable. The magnification for D240 (20 and 100X) was chosen in order to emphasise that all WM115 do not express this marker. Instead for other lymphoangiogenic markers the magnification used $(100 \mathrm{X})$ allowed better visualisation of the presence of these markers (VEGF-R3, prox-1, LYVE-1, podoplanin). The figure shows a representative experiment out of the four carried out. 



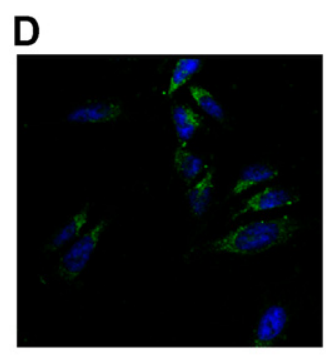

in vitro



tumor xenograft

Fig. 6 - Tumour xenografts of WM115 in NOD-SCID mice and expression of CD133 by flow cytometry, Western blot, RT-PCR and immunofluorescence. 500,000 cells were injected subcutaneously into NOD-SCID mice and the tumours were analysed after 60 days (five animals). (A) A representative flow cytometry analysis. Tumours were incubated for $2 \mathrm{~h}$ in medium containing $\mathbf{0 . 2} \%$ of collagenase type I and single cells were fixed in iced ethanol and stained with anti CD133 PE-conjugated (Myliteni) (red line). The same analysis was performed on WM115 cells grown in vitro (green line). (B) $50 \mu \mathrm{g}$ of tumour xenograft lysates (lane 1) or WM115 cells (lane 2) were loaded on 10\% SDS-PAGE and the antigen-antibody complex was detected with ECL system. Beta-actin was used as housekeeping gene. (C) Level of expression of CD133 by RT-PCR in WM115 cells (lane1), in tumour xenograft (lane 3) and in single cells isolated by tumour xenograft and maintained in vitro for 2-3 passages (lane 2). Total RNA was extracted from WM115 cells using the RNeasy Mini Kit. To exclude potential genomic DNA contamination, samples were treated with RNase-free DNase I and then complementary DNA (cDNA) was synthesised. A band of $470 \mathrm{bp}$ was obtained and sequenced. GAPDH was used as housekeeping gene. (D) Immunofluorescence analysis of CD133 in WM155 cells (in vitro) and in tumour xenograft. Single cells obtained as described above were immediately fixed with 4\% paraformaldehyde for $20 \mathrm{~min}$ and incubated with anti-CD133 (1:100, Abcam). The nuclei were stained with DAPI. All together the data shows that in vivo the WM115 cells express lower levels of CD133 than in vitro.

show different genetic and biological characteristics from which they were derived. The injection of 5000 cells produced a tumour of 350-380 mg in weight after 4 months while $5 \times 10^{5}$ cells gave a tumour of the same weight 2 months after. The level of expression of CD133 was investigated by means of FACS, RT-PCR, Western blot and immunofluorescence. Fig. 6 clearly shows a decreased expression of CD133 in tumor xenograft. Interestingly, even ABCG2 level was lower in respect to WM115 cells grown in vitro (Fig. 7, panel A). By contrast, WM115 cells did not express ABCB1 either in vitro or in vivo (not shown). Accordingly, WM115 cells were negative for rodhamine up-take while in tumour xenograft, $20 \%$ of positive cells were counted (Fig. 7, panel B).

In order to investigate whether the in vivo decrement of both CD133 and ABCG2 expression was reversible under culture conditions, melanoma cells isolated from tumour xenograft were cultured for 2-3 passages and then analysed for the expression of these markers by RT-PCR. As shown in Figs.
6 (panel C) and 7 (panel A), both CD133 and ABCG2 markers were re-expressed.

\subsection{Expression of CD20, CD133 and ABCG2 in spheroid WM115 cells}

A recent paper has shown that WM115 cells propagated as non-adherent spheres (melanoma spheroid cells) expressed higher levels of CD20 in comparison to WM115 adherent cells. Melanoma spheroid cells appeared to be more tumourigenic when grafted into mice. ${ }^{25}$ Thus we have investigated by flow cytometry the expression of CD133 and ABCG2 in adherent and spheroid WM115 cells in comparison to CD20. According to a previous paper, WM115 cells did not express CD20 when grown as adherent cells in vitro, while the melanoma spheroids were enriched in such a factor (data not shown). Interesting, the expression of CD133 and ABCG2 was significantly lower in melanoma spheroids cells than in their adherent 

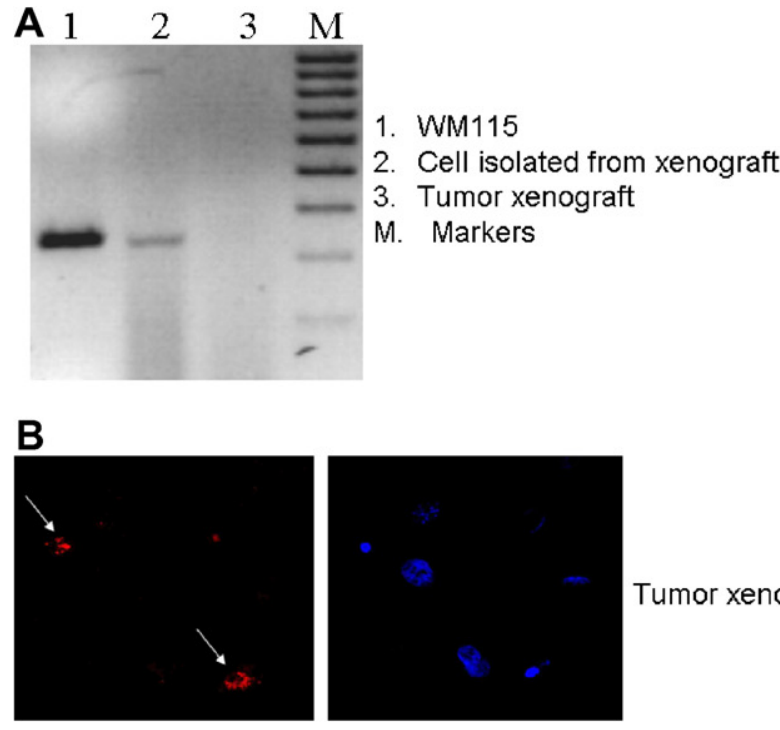

Tumor xenograft
C

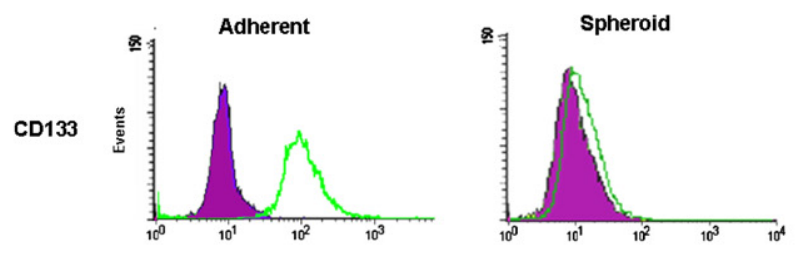

$\mathrm{D}_{\mathrm{CD} 133}$

Log FITC

D133



Fig. 7 - Levels of expression of ABCG2 in WM115 cells and tumour xenograft. Panel A. RT-PCR of ABCG2 in WM115 cells (lane 1), in tumour xenograft (lane 3 ) and in single cells obtained by tumour xenograft and maintained in vitro for 2-3 passages (lane 2). 500,000 WM115 cells were injected subcutaneously into NOD-SCID mice (five animals) and the tumours were analysed after 60 days. Total RNA was extracted from WM115 cells using the RNeasy Mini Kit. To exclude potential genomic DNA contamination, samples were treated with RNase-free DNase I and then complementary DNA (cDNA) was synthesised. A band of $347 \mathrm{bp}$ was obtained and sequenced. GAPDH was used as housekeeping gene. Panel B. Rhodamine uptake in WM115 melanoma cells grown in vivo in NOD-SCID mice (arrows). 500,000 cells were injected subcutaneously into NOD-SCID mice and the tumour was resected after 60 days. Single cells were obtained and incubated with $0.2 \%$ of collagenase type I and maintained in growth medium for a night. Then the cells were incubated with rhodamine $(0.1 \mu \mathrm{g} / \mathrm{ml})$ for $20 \mathrm{~min}$ at $37{ }^{\circ} \mathrm{C}$ and than fixed with $4 \%$ paraformaldehyde for $20 \mathrm{~min}$ at $4{ }^{\circ} \mathrm{C}$. The nuclei were stained with DAPI.These data show lower levels of ABCG2 and higher up-take of rhodamine in tumour xenograft with respect to WM115 cells maintained in vitro. Furthermore, in vitro tumour cells isolated by xenograft re-started to express both CD133 and ABCG2 after few passages in vitro. Levels of CD133 and ABCG2 in WM115 cells and in WM115 spheroids. Panel C. Flow cytometric analysis for CD133 expression on spheroid or adherent cultures of WM115 cells. Fixed cells were incubated with anti-CD133 (epitope 2, CD133/2) (Shaded areas, isotype-matched control; solid lines CD133). The figure shows a typical experiment out of three carried out. No specific staining was evaluated using a monoclonal antibody for IgG1 and IgG2a. Panel D. Total RNA was extracted from WM115 cells using the RNeasy Mini Kit. To exclude potential genomic DNA contamination, samples were treated with RNase-free DNase I and then complementary DNA (CDNA) was synthesised. A band of 347 and 470 bp was obtained for ABCG2 and CD133, respectively and sequenced. GAPDH was used as housekeeping gene.

counterparts (Fig. 7). In contrast, ABCB1 was not expressed (data not shown).

\section{Discussion}

The hypothesis that a tumour may originate from the accumulation of genetic alterations inside immature progenitor/ stem cells can be substantially summarised by three major observations: i) striking phenotypic similarities between normal and CSCs, ii) similar self-renewal potential of stem cell and cancer cells, ${ }^{26,27}$ iii) within the tumour mass only a rare fraction of tumour cells are able to recapitulate the original tumour. ${ }^{26,27}$ In haematological malignancies such as leukaemia, ${ }^{3-5}$ multiple myeloma, ${ }^{26}$ solid tumours such as breast cancer, ${ }^{5}$ and more recently in brain tumours, ${ }^{6,7}$ rare cells were isolated with a remarkable potential of self-renewal and these cells alone were found to drive the formation of the original tumours.

Herein we wanted to investigate whether human melanoma could contain tumour cell subsets that can be referred to as cancer stem/ initiating cells. To this end, we initially analysed the presence of the marker CD133 in fresh human melanoma specimens, because this marker has been found to be expressed in other tumour originating cells. ${ }^{5-7}$

In agreement with the general thinking that cancer stem cells may represent only a very minimal part of the total tumour cell population, we found that CD133 was expressed in a very small percentage of the total melanoma cell population obtained from fresh metastatic specimens. All of the biopsies analysed (nine) never expressed CD133 above 1\% of the total cells examined. Most significantly, only the CD133+ cells, but not their CD133- counterpart of fresh melanoma cells, were able to re-form a melanoma tumour when transplanted in NOD-SCID mice, as confirmed by the histology of the tumour xenograft and the expression of Mart-1, a typical melocytic marker. If confirmed by a larger sample of melanoma biopsies, to our knowledge, this result may be relevant in human melanoma tumour biology. Moreover, it is interesting to note that, like glioblastoma, the tumourigenic potential of melanoma seems to be retained only in cells expressing 
CD133, indicating that these neuroectodermal tumours may originate from a common ancestral progenitor. This hypothesis could be sustained by the evidence that skin derived stem cells can differentiate into neurogenic cell lineages. ${ }^{28}$ Whether other neuroectodermal derived tumours share similar properties remains to be investigated. Since few cells were CD133+ in melanoma biopsy, we analysed the expression of CD133 in a well established human melanoma cell line named WM115 because of its strong tumourigenic potential and their further previous characterisation by our group. ${ }^{21}$ Surprisingly, we found that almost all the WM115 cells expressed CD133 as confirmed by FACS, RT-PCR and immunocytochemistry and they also expressed ABCG2. Thus we hypothesised that this homogeneous expression of CD133 found in practically $95 \%$ of WM115 cells might be the consequence of serial sub-cultivations leading to the rise of selected clones with enhanced stem-ness proprieties. To ascertain this hypothesis, we performed a detailed analysis of WM115 cells that they possess several phenotypic and functional features of stem cells. In particular we found: a) WM115 cells were able to differentiate into neurogenic cell lineages. WM115 cells expressed markers of precursors and mature oligodendrocytes and they are positive for mature neural markers. They did not express GFAP, a marker typical of mature astrocytes. Thus, they showed some features of normal hNSC; ${ }^{6,7}$ b) WM115 cells can differentiate into mesenchymal lineages such as adipogenic cells as we clearly demonstrated by their capacity of incorporating Oil Red and expressing higher levels of adipsin; ${ }^{29}$ c) WM115 cells showed strong similarity to hNSC by their capacity to grow as floating cell aggregates forming spheres under serum free culture conditions. In addition to these typical cancer stem cell features, WM115 showed angiogenic and lymphoangiogenic markers consistent with its high tumourigenic capacity in vivo.

WM115 cell line expresses the main angiogenic factors and in particular notch $4^{30}$ while SOX18, a factor transiently expressed in nascent endothelial cells during embryonic development and adult neovascularisation is undetectable. ${ }^{31}$ Regarding lymphoangiogenic markers, WM115 cells expressed in particular prox-1 which plays a pivotal role in specifying lymphatic endothelial cell fate. ${ }^{20}$ Accordingly, D2-40, an antigen expressed specifically in mature lymphatic vessels $^{32,33}$ was absent. The idea that tumour stem/initiating cells may have the potential to induce angiogenesis and/or lymphoangiogenesis could represent an important new finding suggesting that, besides the typical stem-ness features, cancer stem cells may also possess this angiogenic repertoire in order to recapitulate the original tumour. Whether these properties can be attributed to a multiple interactive cancer stem unit or can be involved in cross-talk interaction with neighbouring host stromal cells remains to be clarified. ${ }^{34}$ The high expression of CD133 on WM115 was in contrast with the low expression of this marker in melanoma cells derived from fresh biopsies. This result thus raises the question if CD133 may represent a good marker for identification of cancer stem cells in a melanoma cell line. However, it was important to note that the expression of CD133 was dramatically reduced in level in fresh melanoma biopsies in the xenograft tumour. In addition, xenograft tumours also showed a signif- icant down modulation of ABCG2, another important marker of cancer stem cells. ${ }^{19,20}$ Thus compared to in vitro, the expression CD133 and ABCG2 on melanoma cells in vivo behaved differently. This observation was confirmed by the result that cultured melanoma cells derived from xenograft after only a few passages re-expressed high level of both CD133 and ABCG2 markers. Therefore, it is tempting to speculate that with CD133+ and ABCG2+ in some tumours, such as melanoma, quiescent stem-like cells present in the tumour mass could be 're-activated' by in vitro conditions becoming the main cellular population after a few passages or could be due to expansion of a rare CD133+ cells. A recent report suggested that WM115 grown as floating cell spheroids were enriched for the expression of CD20, a typical marker of mature B lymphocyte, and this was associated with a higher tumourigenic capacity in vivo. ${ }^{25}$ Thus we have tested the expression of CD133 and ABCG2 in both WM115 spheroids and in adherent WM115 cell cultures. Interestingly, a significant down modulation of these markers was observed in WM115 spheres, suggesting that melanoma cells cultured as floating cell spheroids may behave, at least for the expression of CD133 and ABCG2 markers, more similarly in vivo conditions.

In conclusion, our study showed new important insights into human melanoma. In particular we have identified CD133+ and ABCG2+ as potential melanoma stem/initiating cell markers both in vivo and in vitro. Recently, Lee and colleagues raised the problem that cancer cell lines could not be a good model for studying the cancer stem cell biology of human cancer. $^{35}$ Our findings indicate that WM115 xenografts might be a good model since they showed similar levels of CD133 and ABCG2 detected in biopsy. Another important aspect is the evidence that WM115, a melanoma cell line highly enriched with cells expressing these markers, concomitantly expressed angiogenic and lymphonangiogenic markers, could suggest that, at least in melanoma, cancer stem/ initiating cells may possess on their own many features that are distinctive of more aggressive neoplasma.

\section{Conflict of interest statement}

None declared.

\section{Acknowledgement}

This study was supported by the Italian funding, COFIN.

\section{R E F E R E N C E S}

1. Park $\mathrm{CH}$, Bergsagel DE, McCulloch EA. Mouse myeloma tumor stem cells: a primary cell culture assay. J Natl Cancer Inst 1971;46:411-22.

2. Reya T, Morrison SJ, Clarke MF, Weissman IL. Stem cells, cancer, and cancer stem cells. Nature 2001;414:105-11.

3. Bonnet D, Dick JE. Human acute myeloid leukemia is organized as a heirarchy that originates from a primitive hematopoietic cell. Nat Med 1997;3:730-7. 
4. Passegue E, Jamieson CH, Ailles LE, Weissman IL. Normal and leukemic hematopoiesis: are leukaemias a stem cell disorder or a reacquisition of stem cell characteristics? PNAS 2003;30:S11842-9.

5. Al-Hajj M, Wicha MS, Benito-Hernandez A, Morrison SJ, Clarke MF. Prospective identification of tumorigenic breast cancer cells. PNAS 2003;100:3983-8.

6. Galli R, Binda E, Orfanelli U, et al. Isolation and characterization of tumorigenic, stem-like neural precursors from human glioblastoma. Cancer Res 2004;64:7011-21.

7. Singh SK, Hawkins C, Clarke ID, et al. Identification of human brain tumour initiating cells. Nature 2004;432:396-401.

8. Rizzo S, Attard G, Hudson DL. Prostate epithelial stem cell. Cell Proliferation 2005;38:363-74.

9. De Vries E, Bray FI, Coebergh JWW, Parkin DM. Changing epidemiology of malignant cutaneous melanoma in Europe 1953-1997: rising trends in incidence and mortality but recent stabilizations in western Europe and decreases in Scandinavia. In J Cancer 2003;107:119-26.

10. Barbero A, Ploegert S, Heberer M, Martin I. Plasticity of clonal populations of dedifferentiated adult human articular chondrocytes. Arthritis Rheum 2003;48:1315-25.

11. Jemal A, Murray T, Samuels A, Ghafoor A, Ward E, Thun MJ. Cancer statistics 2003. CA Cancer J Clin 2003;53:5-26.

12. Morton DL, Essner R, Calch C. Surgical excision of distant metastasis. 4th ed. In: Bach C, Houghton A, Sober A, et al., editors. Cutaneous melanoma, 547. St Louis (MO): Quality Medical Publishing; 2003. p. 572-6.

13. Seftor EA, Meltzer PS, Schatteman GC, et al. Expression of multiple molecular phenotypes by aggressive melanoma tumor cells: role in vasculogenic mimicry. Crit Rev Oncol Hematol 2002;44:17-27.

14. Seftor EA, Meltzer PS, Kirschmann DA, et al. Molecular determinants of human uveal melanoma invasion and metastasis. Clin Exp Metastasis 2002;19:233-46.

15. Bittner M, Meltzer P, Chen Y, et al. Molecular classification of cutaneous malignant melanoma by gene expression profiling. Nature 2000;406:536-40.

16. Grichnik JM, Burch JA, Schulteis RD, et al. Melanoma, a tumor based on a mutant stem cell? J Investigative Dermatology 2006;126:142-53.

17. Hemmati HD, Nakano I, Lazareff JA, et al. Cancerous stem cells can arise from pediatric brain tumors. PNAS 2003;100:15178-83.

18. Florek M, Haase M, Marzesco AM, et al. Prominin-1/CD133, a neural and hematopoietic stem cell marker, is expressed in adult human differentiated cells and certain types of kidney cancer. Cell Tissue Res 2005;319:15-26.

19. Zhou S, Schuetz JD, Bunting KD, et al. The ABC transporter Bcrp1/ABCG2 is expressed in a wide variety of stem cells and is a molecular determinant of the side-population phenotype. Nat Med 2001;7:1028-34.

20. Doyle LA, Ross DD. Multidrug resistance mediated by the breast cancer resistance protein BCRP (ABCG2). Oncogene 2003;22:7340-58.
21. La Porta CA, Porro D, Comolli R. Higher levels of melanin and inhibition of cdk2 activity in primary human melanoma cells WM115 overexpressing nPKCdelta. Melanoma Res 2002;12:297-307.

22. Dirks WG, Faehnrich S, Estella IA, Drexler HG. Short tandem repeat DNA typing provides an international reference standard for authentication of human cell lines. ALTEX 2005;22:103-9.

23. Towbin H, Staehelin T, Gordon J. Electrophoretic transfer of proteins from polyacrylamide gels to nitrocellulose sheets: procedure and some applications. PNAS 1979;76:4350-4.

24. Vescovi AL, Parati EA, Gritti A, et al. Isolation and cloning of multipotential stem cells from the embryonic human CNS and establishment of transplantable human neural stem cell lines by epigenetic stimulation. Exp Neurol 1999;156:71-83.

25. Fang D, Nguyen TK, Leishear K, et al. A Tumorigenic Subpopulation with Stem Cell Properties in Melanomas. Cancer Res 2005;65:9328-37.

26. Uchida N, Buck DW, He D, et al. Direct isolation of human central nervous system stem cells. PNAS 2000;97:14720-5.

27. Corbeil D, Roper K, Weigmann A, Huttner WB. AC133 hematopoietic stem cell antigen: human homologue of mouse kidney prominin or distinct member of a novel protein family? Blood 1998;91:2625-6.

28. Toma JG, Akhavan M, Fernandes KJ, et al. Isolation of multipotent adult stem cells from the dermis of mammalian skin. Nat Cell Biol 2001;3:778-84.

29. Barbero A, Ploegert S, Heberer M, Martin I. Plasticity of clonal populations of dedifferentiated adult human articular chondrocytes. Arthritis Rheum 2003;48:1315-25.

30. Leong KG, Hu X, Li L, et al. Activated Notch4 inhibits angiogenesis: role of beta 1-integrin activation. Mol Cell Biol 2002;22:2830-41.

31. Downes M, Koopman P. SOX18 and the transcriptional regulation of blood vessel development. Trends Cardiovasc Med 2001;11:318-24.

32. Fogt F, Zimmerman RL, Ross HM, Daly T, Gausas RE. Identification of lymphatic vessels in malignant, adenomatous and normal colonic mucosa using the novel immunostain D2-40. Oncol Rep 2004;11:47-50.

33. Fogt F, Pascha TL, Zhang PJ, Gausas RE, Rahemtulla A, Zimmerman RL. Proliferation of D2-40-expressing intestinal lymphatic vessels in the lamina propria in inflammatory bowel disease. Int J Mol Med 2004;13:211-4.

34. Rak J. Is cancer stem cell a cell, or a multicellular unit capable of inducing angiogenesis? Medical hypothesis 2006;66:601-4.

35. Lee J, Katlarova S, Katliarov Y, et al. Tumor stem cells derived from glioblastomas cultured in bFGF and EGF more closely mirror the phenotype and genotype of primary tumors than do serum-cultured cell lines. Cancer Cell 2006;9:391-403.

36. Cavazzin C, Ferrari D, Facchetti F, et al. Unique expression and localization of aquaporin-4 and aquaporin-9 in murine and human neural stem cells and in their glial progeny. Glia 2006;53:167-81. 